\title{
TIME
}

B R I L L

Timing \& Time Perception 5 (2017) 280-296

$\overline{\text { brill.com/time }}$

\section{The Temporal Dimensions in the First Year of Life}

\author{
Maria Dolores de Hevia ${ }^{1,2, *}$, Yu-Na Lee ${ }^{3}$ and Arlette Streri ${ }^{1}$ \\ ${ }^{1}$ Université Paris Descartes, Sorbonne Paris Cité, Paris, France \\ ${ }^{2}$ Laboratoire Psychologie de la Perception, UMR 8242 CNRS, 45 rue des Saints-Pères, \\ 75006 Paris, France \\ ${ }^{3}$ Massachusetts Institute of Technology, Cambridge, MA 02139, USA \\ Received October 31, 2016; accepted February 28, 2017
}

\begin{abstract}
Time is a multifaceted concept that is critical in our cognitive lives and can refer, among others, to the period that lapses between the initial encounter with a stimulus and its posterior recognition, as well as to the specific duration of a certain event. In the first part of this paper, we will review studies that explain the involvement of the temporal dimension in the processing of sensory information, in the form of a temporal delay that impacts the accuracy of information processing. We will review studies that investigate the time intervals required to encode, retain, and remember a stimulus across sensory modalities in preverbal infants. In the second part, we will review studies that examine preverbal infants' ability to encode the duration and distinguish events. In particular, we will discuss recent studies that show how the ability to recognize the timing of events in infants and newborns parallels, and is related to, their ability to compute other quantitative dimensions, such as number and space.
\end{abstract}

Keywords

Time, quantity, sensory modes, magnitude, infants, newborns

\section{Measures of Time in Newborns and Infants}

Time is a critical component in our cognitive lives that, together with space and quantity, is considered a requirement of the central nervous system (e.g., Meck \& Church, 1983; Walsh, 2003). Moreover, some have argued that time is the dominant dimension, being virtually impossible to ignore, as this aspect is more salient and dominant than other aspects of received stimulation (Navon, 1978). In the present paper, we will consider two concepts of the dimension of time present

\footnotetext{
* To whom correspondence should be addressed. E-mail: dehevialola@gmail.com
} 
in infancy during the first year of life. On the one hand, time will be discussed in terms of the elapsed temporal period between the initial encounter with a source of stimulation, in its different sensory modalities (e.g., auditory, tactile), and its impact on newborns' and infants' encoding, maintenance, and later recognition of the same stimulation. We will discuss studies for each sensory modality separately. On the other hand, we will review evidence for infants' and newborns' ability to encode and discriminate different temporal durations (i.e., the specific duration of a certain event), and how this ability relates to the discrimination of other quantities such as spatial extent and numerosity. In this second part of the paper, mainly recent studies investigating the ability to create mappings between these dimensions, as well as how the respective developmental courses overlap, will be discussed.

All processes, whether perceptual, motor or cognitive, involve the dimension of time. In adults, time processing is studied with the mental chronometry paradigm, which measures the elapsed time between the presentation of a sensory stimulus and the subsequent behavioral response (Jensen, 2006). Behavioral response time (RT), typically indicated by a button press or vocal response, is an index of processing speed. There have been reports on correlations between behavioral RT and level of intelligence (see Jensen, 1993; Spearman, 1924, 1927), as well as between mental chronometry and memories (Collins \& Quillian, 1969). With the improvement in mental chronometric methods, reaction time to the scale of few hundred milliseconds can be detected from a small millimeter range, providing more detailed understanding to time processing (Formisano \& Goebel, 2003; Posner, 2005). Although this paradigm is considered very difficult to use in infancy, it is not quite impossible. For example, as fetuses are capable of hearing sounds from outside the womb, such as speech and music, researchers are able to investigate auditory attention and behavioral changes by measuring the variations in the cardiac rhythm (Kisilevsky et al., 2004). For instance, by analyzing the spontaneous motor tempo (SMT) of non-nutritive sucking, Bobin-Bègue et al. (2006) observed that infants at the age of two months accelerated their sucking tempo, adjusting to the perceived external tempo that was faster than their endogenous tempo. Similarly, as newborns are capable of visually following a target, we can measure the number and direction of saccades, the number, location, and duration of fixations, as well as the amount of time the newborns spend following the target (see Streri et al., 2013, for a review on newborns' cognitive abilities).

The understanding of the temporal dimension contributes to higher cognitive skills and memories, which shape knowledge, behaviors, choices, and personalities in adulthood. For example, the newborns' preference for their mother's voice, which they remember from the womb, reinforces the bond between newborns and their mother (DeCasper \& Fifer, 1980; DeCasper \& Sigafoos, 1983). In fact, the ability to recognize and remember an object in a new environment is one of 
the most important cognitive functions in humans. Do infants, postnatally or even prenatally, perceive and retain information during a certain duration? How can researchers measure the time lapse between learning and recognition in the various sensory modalities? Several studies have revealed a number of sensory and cognitive abilities in infancy through different methods such as habituation/dishabituation and familiarization (Fantz, 1964; see Colombo \& Mitchell, 2009, for a review), sucking (Haith, 1966), preferential choice between two stimuli (Fantz, 1958), training, and conditioning (see Gottlieb \& Krasnegor 1985). In what follows, we will discuss studies using these different paradigms.

Carolyn Rovee-Collier (Rovee-Collier \& Fagen, 1981; Rovee-Collier \& Hartshorn, 1999) was a pioneer in the study of memory development in young infants. In her research, she typically used a mobile task with two- to six-month-olds, where infants had to learn to move a mobile crib by kicking the foot with a ribbon connected to the crib. In the first phase, the kicking rate produced by infants served as a baseline for comparison with the kicking rate during the second phase. The second phase consisted of the recognition test during which the ribbon was disconnected from the ankle. If the infants recognize the mobile crib, they should kick above the baseline rate; otherwise, the kicking rate at test would not differ from the baseline. The general result from this type of studies is that the duration of memory becomes longer with age. However, results vary according to two parameters: the delay between the two phases and the duration of the training phase. In fact, memory performance can be altered by changing the parameters of training and by manipulating the delay for recovery, regardless of the age of the baby. However, this paradigm does not suit some sensory modalities, such as olfaction, tactile, and even auditory perception.

The paradigm of habituation/dishabituation with an infant-controlled procedure (Horowitz et al., 1972) is a powerful tool, not only because it allows measurements in both learning and recognition abilities from birth onwards, but also because this paradigm can be used with any of the sensory modalities. All studies using a habituation/dishabituation paradigm are based on the assumption that stimulus discrimination and sensory recognition/memory can be inferred from infants' performance when they respond differentially to stimuli that are novel than to those that are familiar. Moreover, it is now well established that the process of habituation and that of memory are narrowly linked, and that they both reveal a form of mental representation of the stimuli presented (e.g., Bornstein, 1985; Pascalis \& de Haan, 2003).

In the first phase of habituation, a stimulus in a certain sensory modality is repeatedly presented to the infants, until a predetermined criterion is reached. The decrease in response to the sensory modality and the stimulus over time indicates decreased attention. This results from the infant's ability to match the stored memory of the previously presented stimulus with the same stimulus presented again. In the second phase, a novel stimulus is presented to the infants in the same 
modality with the expectation of a dishabituation or novel reaction. The reaction to novelty results from a mismatch between the newly presented stimulus and the memorized representation. Between these two phases, a delay or temporal 'window' can be inserted in order to test the strength of the memory (see Oakes, 2010, for a review). In the first part of this paper, we characterize the sensory temporal windows in different modalities.

\section{The Sensory Temporal Windows of Time in Infancy}

\subsection{Visual Memory in Infancy}

The visual sensory mode is considered a significant modality for humans due to the great amount of visual information that humans receive from the environment. However, humans' visual modality functions only after birth and not in utero like other sensory modes such as auditory, tactile, and olfactory that are already functional during the prenatal period. Studies using the habituation/dishabituation procedure were the first to show that infants have visual memories for several patterns already at birth (Friedman \& Carpenter, 1971; Friedman et al., 1970; see also Slater et al., 1982). Some studies have focused on the duration of time from the presentation of a visual stimulus to its recognition. There have been studies on face memory, specifically on newborns' recognition memory of the mother's face. In fact, several studies on four-day-old newborns have demonstrated that there exists a preference for the mother's face compared to a stranger's face (Bushnell et al., 1989; Field et al., 1984). In another study by Pascalis et al. (1995), similar results have shown four-day-old newborns' preference for the mother's face over an unknown yet similar looking woman's face, even after a three- to four-minute delay in time from when the mother was out of sight to the time when the mother reappeared as a part of the study's visual stimulus. The duration of time indicated that the newborns did not use transitional memory, but recognition memory (see also Pascalis \& de Schonen, 1994). For newborns right after birth, bimodal stimulation allowed the recognition memory interval to extend to about seven hours as shown in Sai's (2005) study. Mothers' verbal interactions with their newborn, in addition to the visual presentation, helped improve the memorization of the mother's face compared to having only visual interaction alone. This study also reveals that multisensory perception is crucial for the development of social and cognitive abilities from birth.

\subsection{Haptic Memory in Infancy}

Studies on the adult haptic system without visual cues show that the manual haptic system gathers a relatively wide range of information regarding the observed objects' properties and depends on specific exploratory procedures for each property. For instance, unsupported holding is used to process the weight of an object, whereas lateral motion is used to process the texture of an object. 
(Lederman \& Klatzky, 1987). On the other hand, observations on newborns using habituation/dishabituation with both the left and the right hands suggest that the haptic memory, present from birth, does not depend on specific exploratory procedures (Streri et al., 2000). Indeed, from birth, infants are capable of encoding, holding in memory, and recognizing a certain amount of information gathered on various object properties, such as shape, substance, weight, size, and volume. All these properties are gathered through an exploratory procedure, such as the enclosure procedure by means of exerting some light grasps on the objects.

Haptic memory in young infants has been studied as well. Lhote and Streri (1998) showed that infants are able to manually recognize an object after a 30 -second interval by two months of age and after a two-minute interval (longterm memory) by four months of age (Lhote \& Streri, 2003). Nevertheless, haptic recognition is often sensitive to haptic interference.

Gottfried and Rose (1980) were the first to study the haptic recognition memory in one-year-old babies. In their study, infants responded differently, depending on the degree of familiarity or novelty of an object, showing signs of recognition memory. After a two-minute period of haptic familiarization without visual control, the young infants manipulated novel objects differently from familiar objects. Few additional studies have looked into infants' capability to retain information from the haptic memory. Catherwood (1993) tested the robustness of the haptic memory on shape and texture in eight-month-old babies. The babies attested to good texture memory and showed recognition of the shapes of familiar objects after a five-minute break, but not after a haptic interference (presentation of a new object). This failure happened due to the short duration of the familiarization phase, which was limited to 30 seconds of manipulation or contact. As for the conditioning paradigm, haptic memory depends on the duration of familiarization as well as on the recovery delay.

\subsection{Auditory Memory in Newborns}

As previously mentioned, auditory perception is present during the fetal period. Consequently, newborns' auditory memory is considered quite remarkable for, among others, a critical reason: both newborns and older infants have to perceive, identify, understand, produce, and learn speech. In order to achieve this, newborns have to segment speech in significant units, such as words. In fact, evidence shows that long-term auditory memory exists in newborns. Swain and colleagues (1993) compared the response rates between two groups of newborn infants that were presented speech sounds in two sessions, which were separated by a time interval of 24 hours. Habituation and recovery were assessed by observing the spontaneous movement of the head orienting according to the sound's location. Results showed that infants who heard the same word in both sessions were less responsive than infants who heard different words. Another study using habituation and heart rate response to auditory stimuli showed a similar result after a 
24-hour delay but not after a five-day delay (Graham \& Jackson, 1970). Spence (1996) examined preferences for a novel or familiar melody by observing the nonnutritive sucking rate of one- to two-month-olds. After multiple familiarization sessions, the test phases occurred during each of three days. The following results were observed: on day one of the retention interval, a novelty preference was observed; on day two, no consistent preference occurred; and on day three, a preference for the familiar melody was observed. This preference pattern reflects on the development of representation of the long-term memory. According to Hunter and Ames' model (1988), the longer the interval between the stimulus presentation and the recognition test, the weaker the representation of the memorized stimulus. As a consequence, longer intervals produced a higher discrepancy between the internal representation and the external stimulus, and, therefore, a familiarity preference was observed. Bahrick and Pickens (1995) found a similar pattern of preference, where infants preferred novel motion after a short retention period (one minute after the presentation) but familiar motion after a longer retention period (periods of one and three months).

Several studies have reported a retention period over a much longer time extending across the prenatal-postnatal boundary (DeCasper \& Fifer, 1980; DeCasper \& Spence, 1986; Fifer \& Moon, 1994; Moon \& Fifer, 1990). These authors have demonstrated that newborns prefer their mothers' voices to female strangers' voices or to melodies sung by the mother during the prenatal period. Moreover, this preference persists for several days up to one month after birth.

\subsection{Olfactory Memory in Newborns}

As for the auditory modality, chemical senses, such as olfaction and taste, are already functional in utero. The preferential choice between two odors is the more often utilized procedure to test infants' olfaction abilities. Schaal (2005) has examined the role played by olfaction in newborns' behavior during the transition from the prenatal to the postnatal environment. This author considers that prenatal olfactory experiences strongly influence newborns' postnatal orientation to the odor environment. For example, newborns are attracted to the odor of their own amniotic fluid when it is presented alone, as well as when presented in a doublechoice test (Marlier et al., 1998). However, two days after birth, the newborn treats in an equivalent manner both the odor of the amniotic fluid and the mother's colostrum. It is by the fourth day that infants orient their head for a longer duration toward their mother's milk odor compared to the odor of their own amniotic fluid. These findings are in line with the hypothesis of a progressive shift and adaptation of the infant's perception of the odor quality of milk relative to that of amniotic fluid. In summary, these results reinforce the transnatal chemosensory continuity in mammals, by which perinatal substrates carry odor information with similar chemosensory and/or motivational characteristics, and, therefore, an inability to discriminate between them is present early in postnatal life. 
Other authors found the existence of a sensitive period for olfactory learning in human newborns (Romantshik et al., 2007). Two groups of newborns were exposed to an odorant for 30 min either 4-37 minutes after birth (early exposure) or $12 \mathrm{~h}$ postpartum (late exposure). Several days later, newborns' head orientation responses to the familiar as compared to the unfamiliar odor were tested. Newborns in the early-exposure condition spent more time oriented towards the familiar odor than towards the novel, but newborns in the late-exposure condition did not show any difference. This finding supports the idea of a short sensitive period of odor learning in newborns.

\subsection{Final Remarks}

To summarize, although all of the sensory modalities are able to receive, encode, and retain information, the strength of the created memories differs between them. One possible account for this contrasting pattern is that the sensory modes do not synchronously develop during the fetal period. The tactile system starts developing first, followed by the taste and olfactory systems, and, finally, the auditory system. Although the visual mode is formed in utero, its efficiency is only present post birth (see Bremner et al., 2012). This developmental scenario contrasts with the memory performances of each sensory mode. In particular, tactile memory in very young infants seems to be less efficient than other modalities. A plausible explanation is that it is necessary for infants to establish frequent manual contact with objects in order to process efficiently the information, and this is in place from four to five months of age, when the coordination between vision and prehension is possible. Moreover, there is an inherent difficulty in the manipulation of objects by very young infants because of immature motor abilities. In contrast, in the continuous perception of the visual and olfactory modalities the information taken in by these modalities seems to be received 'passively' from the environment. The auditory modality is evidently very efficient at birth because receptors are well mature in utero. This early maturity of audition allows young infants to finely perceive speech and later learn to speak, which links to language, one of the most fundamental cognitive functions in human beings. However, these divergences in the practice of each sensory mode come from their specificities to process information from the environment. Despite these differences and the specificity of information that each sensory mode is able to process, links between modalities and multisensory integration are nevertheless possible (see Streri, 2012).

\section{The Representation of Time in Infants and Newborns}

Although little research has addressed the early development of timing abilities in humans, the last few years evidence has been provided for the representation of the dimension of 'time' by preverbal infants, which is considered one of the 
precursors of mature computational abilities (see Allman et al., 2012, for a review). Studies investigating human infants' ability to time events in their environment have shown that it develops very early in life and is present even from birth. The measure of time considered in studies investigating infants' ability to time events usually refers to the duration of an event that is commonly delivered in the auditory or the audiovisual modalities (e.g., the duration of a tone, or the duration of a visual object that is coupled with a sound). The interest in understanding the infants' ability to process time has taken place in the context that considers the dimensions of time, number, and space, three environmental attributes used by animals and humans to represent the world (Gallistel, 1990; Meck \& Church, 1983).

Research on animals and humans investigating the ability to time events has revealed that temporal discrimination depends on Weber's law: discrimination of two temporal events depends on the ratio between the two values, not on their absolute difference (Meck, 2003). Thus, discriminating between $1.5 \mathrm{~s}$ and $3 \mathrm{~s}$ is equally difficult as discriminating between $3 \mathrm{~s}$ and $6 \mathrm{~s}$, even though the absolute difference in the two pairs differs. This processing signature characterizes discrimination performance in many perceptual dimensions, such as number (Van Oeffelen \& Vos, 1982), spatial extent (Johnson, 1939), brightness and loudness (Stevens \& Marks, 1965), and even abstract dimensions such as the ferocity or the intelligence of animals (Banks \& Flora, 1977). Therefore, a main cognitive attribute characterizing representations of quantitative dimensions is their analog format.

During the first year of life, infants are able to discriminate two event durations. For instance, infants are able to discriminate the two different temporal durations of two audiovisual events created by a puppet coupled with a tone (vanMarle \& Wynn, 2006). Critically, for infants aged six months, a $1: 2$ ratio difference between the two durations is needed in order for discrimination to take place, as at this same age a $2: 3$ ratio difference does not warrant successful discrimination (vanMarle \& Wynn, 2006). For instance, infants habituate to an event duration of $2 \mathrm{~s}$ and are then tested with the same event that lasts for either $2 \mathrm{~s}$ in some trials or $4 \mathrm{~s}$ in other trials. If infants are able to recognize the test duration that is equal to the habituated one, they should show increased attention to the trials containing the novel event duration. This is precisely what was observed in vanMarle and Wynn's study (see also Brannon et al., 2007, for similar findings at six months of age). In contrast, in this same study it was observed that when the events to be discriminated differed by a $2: 3$ ratio ( 3 vs. $4.5 \mathrm{~s}$, or .67 vs. $1 \mathrm{~s})$ infants failed to show any discrimination at test. However, the precision with which temporal events are encoded and remembered improves with age. In fact, approximately four months later, infants are able to discriminate the duration of two events when these differ by a $2: 3$ ratio. When 10-month-old infants are tested on their ability to discriminate between two events lasting for 2 as compared to $3 \mathrm{~s}$, the differing looking patterns at test reflect an increased attention to the 
novel duration (Brannon et al., 2007). Infants' acuity for duration discrimination at 10 months of age allows them to discriminate two durations that differ, at least, by a $2: 3$ ratio, as reported by using different absolute values (i.e., 1 vs. $1.5 \mathrm{~s}$ and 2 vs. $3 \mathrm{~s}$ ). They fail, however, at discriminating durations that differ by a smaller ratio, $3: 4$, suggesting that this ratio is approaching the upper limit of their capacity (Brannon et al., 2007). Therefore, during the first year of life there is a significant improvement in the acuity to represent and discriminate temporal duration.

The developmental trajectory in temporal discrimination parallels the ones described for discrimination of numerical information and spatial extent in preverbal infants: at six months of age, infants need a $1: 2$ ratio to discriminate between two different numerosities (Lipton \& Spelke, 2003; Xu \& Spelke, 2000), or between the size of two objects (Brannon et al., 2006); for both dimensions, a $2: 3$ ratio ensures discrimination at 9nine months of age (Lipton \& Spelke, 2003; Brannon et al., 2006; see Feigenson, 2007, for a review). The parallelisms in the precision with which infants represent magnitude changes in the domains of number, area or spatial extent, and time, support the idea that magnitude representations may be rooted in a single developmental algorithm for 'more than/less than' distinctions (for reviews, see Bonn \& Cantlon, 2012; Cantlon et al., 2009; de Hevia, 2016; Feigenson, 2007). This idea has been inspired by the seminal studies on the common representation of time and number in rats (Meck \& Church, 1983), suggesting that the same mental mechanism underlies the discrimination of these dimensions: the accumulator model proposed by Meck and Church posits that a single mechanism ('accumulated magnitude') is used for both counting and timing, and that there is quantitative equivalence between an estimate of number and a unit of time (Meck et al. 1985).

\subsection{Links between Time, Space, and Number}

Interest in the links between the dimensions of space, time, and number has a rather long tradition. Philosophers like Kant (1781/2010) and psychologists like Piaget $(1952,1969)$ noted the deep cognitive correspondences between these dimensions. For instance, Piaget (1952) described that in the number conservation task, where the experimenter shows two horizontal rows of tokens in oneto-one correspondence, three- to six-year-old children judge longer rows as being greater in number even when they have fewer tokens. Moreover, they think that the number of objects in a row increases as the experimenter spreads the objects apart. Piaget (1969) also argued that in young children temporal judgments are not distinct from spatial judgments: temporal judgments of 'earlier' and 'later' or comparisons of temporal durations such as 'shorter' and 'longer' are all based on spatial distance.

Some authors have argued that the cognitive (and neural) links between the dimensions of space, time, and number (quantity) reflect the existence of a single analog magnitude system that represents all these dimensions under the 
same format (Bueti \& Walsh, 2009; Lourenco \& Longo, 2010; Walsh, 2003). Other authors have claimed, however, that the links between these different dimensions relate to theories of metaphorical mental representation (Lakoff \& Johnson, 1999), by which abstract concepts such as time would use a more concrete dimension built through perceptuo-motor experience - such as the dimension of space - for the purpose of representation. This theory is based on the assumption that metaphoric construction processes are mediated by learning to flexibly apply spatial words for different non-spatial concepts. However, during the gestational period human fetuses are exposed to temporally structured (e.g., mother's heartbeat) information much before they can use visual spatial information, undermining the view that time perception develops upon space. For example, fetal MEG studies have shown that fetuses are sensitive to an auditory-temporal expectancy violation (Sheridan et al., 2010, for a review), as are preterm infants who show temporal discriminative abilities at birth although they are not supposed to use visual information at this time (see Mento \& Bisiacchi, 2012, for a review). The asymmetric relationship between the dimensions of space and time observed in linguistic expressions as well as in behavioral tasks during childhood and adulthood (Casasanto \& Boroditsky, 2008; Casasanto et al., 2010) might depend upon the availability of language and cultural artifacts, as both preverbal infants and nonhuman animals show a symmetric link between these dimensions (Lourenco \& Longo, 2010; Merritt et al., 2010). In fact, research on human infants has revealed in the past few years that preverbal humans do map representations of space, time, and number to one another during their first year of life, before using language, and even a few hours after birth.

In particular, one set of experiments investigating the developmental origins of metaphorical thinking has shown that at nine months of age infants are able to learn a mapping rule that establishes a positive relationship between space and time (e.g., the bigger the space, the longer the time) but fail at learning a negative relationship (e.g., the bigger the space, the shorter the time; Srinivasan \& Carey, 2010). The authors argue that the functional link between the representations of spatial extent and temporal duration does not reflect metaphoric thought but rather may derive from an evolutionary recycling of spatial representations for more general purposes. These links, or mappings, between the dimensions of spatial extent and temporal duration by infants during the first year of life extend to the numerical domain. In particular, Lourenco and Longo (2010) showed that at nine months of age infants are able to generalize an arbitrary rule from one domain to the other. For instance, when habituated to the rule establishing that 'many' objects are colored in black and 'few' objects are colored in white (number domain), the infants look longer to test trials that violate this rule in the temporal domain (i.e., they recover attention with 'black' objects that last for a short duration). This study showed that infants are able to generalize bidirectionally across the three dimensions, spatial extent, temporal duration, and numerosity. 
Finally, a third study using two different methods showed that eight-month-old infants spontaneously create number-space mappings (de Hevia \& Spelke, 2010). In particular, this study showed that: 1) when infants are given congruent numberlength pairings during habituation, where larger numbers are paired with longer lines, and are then presented with new pairings that follow the same (congruent) or a new (incongruent: where larger numbers are paired with shorter lines) rule, they look preferentially at familiar pairings at test (i.e., that follow the same congruent rule);2) when infants are given incongruent number-length pairings during habituation, and are then presented with new pairings that follow the same (incongruent) or a new (congruent) rule, they do not show any preferential looking pattern; 3 ) infants are able to generalize the habituation order (i.e., increasing vs. decreasing) from the numerical domain to the spatial domain. These studies show that the relationship between number, duration, and length, has a privileged structure, with larger durations corresponding to greater horizontal lengths and larger numbers for infants, as for adults (Casasanto \& Boroditsky, 2008; de Hevia \& Spelke, 2009; de Hevia et al. 2006, 2008; Srinivasan \& Carey, 2010; Xuan et al., 2007).

Recent research has addressed the possibility that these associations are the result of experience with the environment, where correlations between space, time, and number occur naturally. In fact, the above reviewed studies could be accounted for by the observation during the first years of life that these magnitudes are usually paired in a congruent way. Studies with newborns tested a few hours after birth, so that experience is at least minimized, can offer some hints to this question. In a study using a familiarization method, one- to three-day-old infants were presented simultaneously with auditory sequences (containing either information of number alone, of duration alone, or both sources of information) and visual line lengths, and their looking times were then measured in new trials containing both novel and familiar information. It was found that when the numerosity and/or duration of the auditory sequence increased from familiarization to test (e.g., from six syllables to 18 syllables), newborns expected the visual length also to increase; when the auditory numerical and/or temporal information decreased (e.g., from a long tone to a short tone), they expected the visual length to decrease. These findings show that newborns create magnitude-congruent expectations across the different dimensions. These expectations, as found for older infants, obey a fixed, congruent structure: when the changes in magnitude across the dimensions vary in opposite directions (one increases, the other decreases), newborn infants do not react to these displays (de Hevia et al., 2014). These findings suggest that these mappings are given by an early predisposition to relate these quantitative dimensions and not to a learning period following the (extensive) exposure to the natural correlations between these variables in the environment, as infants were tested a few hours after birth.

Although this study suggests that the origins of these mappings are not found in enculturation or extensive learning and experience, they leave open the 
question of the functional origins of the early ability to related time, space, and number. It might result from a single-purpose system of magnitude that operates on these (and possibly other) dimensions that generates an undifferentiated representation of 'more than' and 'less than'; alternatively, there might be distinctive representations for each domain that, although sharing a similar format as well as anatomical connections, are fundamentally separate. Some work with infants during the first year of life has shown that at eight months of age infants are not able to successfully create number-brightness pairings, for instance, and that their performance in these tasks differs crucially from their performance in number-length mappings (de Hevia \& Spelke, 2013). Again, experience during the first years of life could account for these findings if number-length pairings are somehow privileged in the environment. Studies with newborns testing pairings between different quantity dimensions could shed more light on this question. Another important source of information could be found in the understanding of the neural origins of these dimensions in newborns, looking for similarities and discrepancies in the way the immature brain treats different dimensions.

\subsection{Final Remarks}

The mental representation of abstract concepts through a spatial dimension is a core property of the human mind. In particular, representations in many domains such as emotional valence (positive is up, negative is down; Meier \& Robinson, 2004), social dominance (powerful is up, powerless is down; Schubert, 2005), or number (small numbers are on the left, large numbers on the right; Dehaene et al., 1993), exploit a spatial mental representation. Time is no exception. In many cultures, for instance, the future is mentally pictured ahead and the past behind one's own body (Clark, 1973). As recent research has shown that the spatial properties associated to number are not learned or dependent on language, but inherently related to its meaning (see de Hevia, 2016; Rugani \& de Hevia, 2017, for reviews), future research on human newborns' spatial conception of time could shed much light on how this dimension is structured and reasoned about early in development.

\section{Conclusion}

Despite the vagueness of sensory information about time, this dimension constitutes a relevant property of infants' cognitive lives. We have reviewed evidence showing that for humans in their first year of life, and even at birth, time not only impacts their memory and recognition abilities across sensory modalities, but that infants can create a mental representation of time over which they can operate and create links with other sources of information that convey quantity meaning. Still, it is an open question whether these two operationalizations of the temporal dimension tap onto the same representational system in infancy: are 
both 'times' represented in the same way? The first sense of time, which is related to learning and to the strength of the memories, is a temporal gap during which no stimulation is occurring, while in the second sense of time the duration is linked to a sensory stimulation. In fact, the latter can be mapped onto other quantity representations, but it is unknown whether the first can enter into computations that take into account the specific duration of the gap. Future research could address this question in preverbal infants.

\section{Acknowledgements}

The authors wish to thank the reviewers for their suggestions. MDdH was supported by the Fondation Fyssen and the Agence Nationale de la Recherche Scientifique, France (ANR-15-CE28-0003-01 NUMSPA).

\section{References}

Allman, M. J., Pelphrey, K. A., \& Meck, W. H. (2012). Developmental neuroscience of time and number: Implications for autism and other neurodevelopmental disabilities. Front. Integr. Neurosci., 6, 7. doi: 10.3389/fnint.2012.00007.

Bahrick, L. E., \& Pickens, J. N. (1995). Infant memory for object motion across a period of three months: Implications for a four-phase attention function. J. Exp. Child Psychol., 59, 343-371.

Banks, W. P., \& Flora, J. (1977). Semantic and perceptual processes in symbolic comparisons. J. Exp. Psychol. Hum. Percept. Perform., 3, 278-290.

Bobin-Bègue, A., Provasi, J., Marks, A., \& Pouthas, V. (2006). Influence of auditory tempo on the endogenous rhythm of non-nutritive sucking. Eur. Rev. Appl. Psychol., 56, 239-245.

Bonn, C. D., \& Cantlon, J. F. (2012). The origins and structure of quantitative concepts. Cogn. Neuropsychol., 29, 149-173.

Bornstein, M. H. (1985). Habituation of attention as a measure of visual information processing in human infants: Summary, systematization, and synthesis. In G. Gottlieb, \& N. A. Krasnegor (Eds), Measurement of audition and vision in the first year of postnatal life: A methodological overview (pp. 253-300). Westport, CT, USA: Ablex Publishing.

Brannon, E. M., Lutz, D., \& Cordes, S. (2006). The development of area discrimination and its implications for number representation in infancy. Dev. Sci., 9, F59-F64.

Brannon, E. M., Suanda, S., \& Libertus, K. (2007). Temporal discrimination increases in precision over development and parallels the development of numerosity discrimination. Dev. Sci., 10, 770-777.

Bremner, A. J., Lewkowicz, D. J., \& Spence, C. (2012). The multisensory approach to development. In A. J. Bremner, D. J. Lewkowicz, \& C. Spence (Eds), Multisensory development (pp. 1-26). Oxford, UK: Oxford University Press.

Bueti, D., \& Walsh, V. (2009). The parietal cortex and the representation of time, space, number and other magnitudes. Phil. Trans. R. Soc. Lond. B Biol. Sci., 364(1525), 1831-1840.

Bushnell, I. W. R., Sai, F., \& Mullin, J. T. (1989). Neonatal recognition of the mother's face. Br. J. Dev. Psychol., 7, 3-15.

Cantlon, J. F., Platt, M. L., \& Brannon, E. M. (2009). Beyond the number domain. Trends Cogn. Sci., 13, 83-91. 
Casasanto, D., \& Boroditsky, L. (2008). Time in the mind: Using space to think about time. Cognition, 106, 579-593.

Casasanto, D., Fotakopoulou, O., \& Boroditsky, L. (2010). Space and time in the child's mind: Evidence for a cross-dimensional asymmetry. Cogn. Sci., 34, 387-405.

Catherwood, D. (1993). The robustness of infant haptic memory: Testing its capacity to withstand delay and haptic interference. Child Dev., 64, 702-710.

Clark, H. H. (1973). The language-as-fixed-effect fallacy: A critique of language statistics in psychological research. J. Verbal Learning Verbal Behav., 12, 335-359.

Collins, A. M., \& Quillian, M. R. (1969). Retrieval time from semantic memory. J. Verbal Learning Verbal Behav., 8, 240-247.

Colombo, J., \& Mitchell, D. W. (2009). Infant visual habituation. Neurobiol. Learn. Mem., 92, 225-234.

DeCasper, A. J., \& Fifer, W. P. (1980). Of human bonding: Newborns prefer their mothers' voices. Science, 208(4448), 1174-6.

DeCasper, A. J., \& Sigafoos, A. D. (1983). The intrauterine heartbeat: A potent reinforcer for newborns. Infant Behav. Dev., 6, 19-25.

DeCasper, A. J., \& Spence, M. J. (1986). Prenatal maternal speech influences newborns' perception of speech sounds. Infant Behav. Dev., 9, 133-150.

Dehaene, S., Bossini, S., \& Giraux, P. (1993). The mental representation of parity and number magnitude. J. Exp. Psychol. Gen., 122, 371-396.

de Hevia, M. D. (2016). Core mathematical abilities in infants: Number and much more. Prog. Brain Res., 227, 53-74.

de Hevia, M. D., \& Spelke, E. S. (2009). Spontaneous mapping of number and space in adults and young children. Cognition, 110, 198-207.

de Hevia, M. D., \& Spelke, E. S. (2010). Number-space mapping in human infants. Psychol. Sci., 21, 653-660.

de Hevia, M. D., \& Spelke, E. S. (2013). Not all continuous dimensions map equally: Numberbrightness mapping in human infants. PLoS One, 8, e81241. doi: 10.1371/journal.pone.0081241.

de Hevia, M. D., Girelli, L., \& Vallar, G. (2006). Numbers and space: a cognitive illusion? Exp. Brain Res., 168, 254-264.

de Hevia, M. D., Girelli, L., Bricolo, E., \& Vallar, G. (2008). The representational space of numerical magnitude: Illusions of length. Q. J. Exp. Psychol., 61, 1496-1514.

de Hevia, M. D., Izard, V., Coubart, A., Spelke, E. S., \& Streri, A. (2014). Representations of space, time, and number in neonates. Proc. Natl Acad. Sci. USA, 111, 4809-4813.

Fantz, R. L. (1958). Pattern vision in young infants. Psychol. Rec., 8, 43-47.

Fantz, R. L. (1964). Visual experience in infants: Decreased attention to familiar patterns relative to novel ones. Science, 146(3644), 668-670.

Feigenson, L. (2007). The equality of quantity. Trends Cogn. Sci., 11, 185-187.

Field, T. M., Cohen, D., Garcia, R., \& Greenberg, R. (1984). Mother-stranger face discrimination by the newborn. Infant Behav. Dev., 7, 19-25.

Fifer, W. P., \& Moon, C. M. (1994). The role of mother's voice in the organization of brain function in the newborn. Acta Paediatr. Suppl., 397, 86-93.

Formisano, E., \& Goebel, R. (2003). Tracking cognitive processes with functional MRI mental chronometry. Curr. Opin. Neurobiol., 13, 174-181.

Friedman, S., \& Carpenter, G. C. (1971). Visual response decrement as a function of age of human newborn. Child Dev., 1967-1973. 
Friedman, S., Nagy, A. N., \& Carpenter, G. C. (1970). Newborn attention: Differential response decrement to visual stimuli. J. Exp. Child Psychol., 10, 44-51.

Gallistel, C. R. (1990). The organization of learning. Cambridge, MA, USA: MIT Press.

Gottfried, A. W., \& Rose, S. A. (1980). Tactile recognition memory in infants. Child Dev., 51, 69-74.

Gottlieb, G., and Krasnesgor, N. (1985). Measurement of audition and vision in the first year of postnatal life. Norwood, NJ, USA: Ablex.

Graham, F. K., \& Jackson, J. C. (1970). Arousal systems and infant heart rate responses. Adv. Child Dev. Behav., 5, 59-117.

Haith, M. M. (1966). The response of the human newborn to visual movement.J. Exp. Child. Psychol., 3, 235-243.

Horowitz, F. D., Paden, L., Bhana, K., \& Self, P. (1972). An infant-control procedure for studying infant visual fixations. Dev. Psychol., 7, 90.

Hunter, M. A., \& Ames, E. W. (1988). A multifactor model of infant preferences for novel and familiar stimuli. In C. Rovee-Collier \& L. P. Lipsitt (Eds), Advances in infancy research, Vol. 5 (pp. 69-93), Norwood, NJ, USA: Ablex.

Jensen, A. R. (1993). Why is reaction time correlated with psychometric g? Curr. Dir. Psychol. Sci., 2, 53-56.

Jensen, A. R. (2006). Clocking the mind: Mental chronometry and individual differences. Amsterdam, Netherlands: Elsevier.

Johnson, D. M. (1939). Confidence and speed in the two-category judgment. Arch. Psychol., 241, 1-52.

Kant, I. (1781/2010). The critique of pure reason (J. M. D. Meiklejohn, transl.), Hazleton, PA, USA: PSU-Hazleton.

Kisilevsky, B. S., Hains, S. M. J., Jacquet, A. Y., Granier-Deferre, C., \& Lecanuet, J. P. (2004). Maturation of fetal responses to music. Dev. Sci., 7, 550-559.

Lakoff, G., \& Johnson, M. (1999). Philosophy in the flesh: The embodied mind and its challenge to western thought. New York, NY, USA: Basic books.

Lederman, S. J., \& Klatzky, R. L. (1987). Hand movements: A window into haptic object recognition. Cogn. Psychol., 19, 342-368.

Lhote, M., \& Streri, A. (1998). Haptic memory and handedness in 2-month-old infants. Laterality, 3, 173-192.

Lhote, M., \& Streri, A. (2003). La mémoire haptique de la forme des objets chez les bébés âgés de 4 mois. Annee Psychol., 103, 33-49.

Lipton, J. S., \& Spelke, E. S. (2003). Origins of number sense: Large-number discrimination in human infants. Psychol. Sci., 14, 396-401.

Lourenco, S. F., \& Longo, M. R. (2010). General magnitude representation in human infants. Psychol. Sci., 21, 873-881.

Marlier, L., Schaal, B., \& Soussignan, R. (1998). Neonatal responsiveness to the odor of amniotic and lacteal fluids: A test of perinatal chemosensory continuity. Child. Dev., 69, 611-623.

Meck, W. H. (Ed.) (2003). Functional and neural mechanisms of internal timing. Boca Raton, FL, USA: CRC.

Meck, W. H., \& Church, R. M. (1983). A mode control model of counting and timing processes. J. Exp. Psychol. Anim. Behav. Process., 9, 320-334.

Meck, W. H., Church, R. M., \& Gibbon, J. (1985). Temporal integration in duration and number discrimination. J. Exp. Psychol. Anim. Behav. Process., 11, 591-597.

Meier, B. P., \& Robinson, M. D. (2004). Why the sunny side is up associations between affect and vertical position. Psychol. Sci., 15, 243-247. 
Mento, G., \& Bisiacchi, P. S. (2012). Neurocognitive development in preterm infants: Insights from different approaches. Neurosci. Biobehav. Rev., 36, 536-555.

Merritt, D. J., Casasanto, D., \& Brannon, E. M. (2010). Do monkeys think in metaphors? Representations of space and time in monkeys and humans. Cognition, 117, 191-202.

Moon, C., \& Fifer, W. P. (1990). Newborns prefer a prenatal version of mother's voice. Poster presented at the biannual meeting of the International Society of Infant Studies, Montreal, QC, Canada.

Navon, D. (1978). On a conceptual hierarchy of time, space, and other dimensions. Cognition, 6, 223-228.

Oakes, L. M. (2010). Using habituation of looking time to assess mental processes in infancy.J. Cogn. Dev., 11, 255-268.

Pascalis, O \& de Haan, M (2003). Recognition memory and novelty preference: What model? In H. Hayne \& J. Fagen (Eds), Progress in infancy research, Vol. 3 (pp. 95-120), Mahwah, NJ, USA: Lawrence Erlbaum Associates.

Pascalis, O., \& de Schonen, S. (1994). Recognition memory in 3- to 4-day-old human neonates. Neuroreport, 5, 1721-1724.

Pascalis, O., de Schonen, S., Morton, J., Deruelle, C., \& Fabre-Grenet, M. (1995). Mother's face recognition by neonates: A replication and an extension. Infant Behav. Dev., 18, 79-85.

Piaget, J. (1952). The child's conception of numbers (C. Gattegno and F. M. Hodgson Transl.). London, UK: Routledge and Kegan Paul (original work published 1941).

Piaget, J. (1969). The child's conception of time (A. J. Pomerans, Transl.). New York, NY, USA: Basic books (original work published 1946).

Posner, M. I. (2005). Timing the brain: Mental chronometry as a tool in neuroscience. PLoS Biol, 3, e51. doi: 10.1371/journal.pbio.0030051.

Romantshik, O., Porter, R. H., Tillmann, V., \& Varendi, H. (2007). Preliminary evidence of a sensitive period for olfactory learning by human newborns. Acta Paediatr., 96, 372-376.

Rovee-Collier, C. K., \& Fagen, J. W. (1981). The retrieval of memory in early infancy. Adv. Infancy Res., $1,225-254$.

Rovee-Collier, C., \& Hartshorn, K. (1999). Long-term maintenance of infant memory. Dev. Psychobiol., 35, 91-102.

Rugani, R., \& de Hevia, M. D. (2017). Number-space associations without language: Evidence from preverbal human infants and non-human animal species. Psychon. Bull. Rev., 24, 352-369.

Sai, F. Z. (2005). The role of the mother's voice in developing mother's face preference: Evidence for intermodal perception at birth. Infant Child Dev., 14, 29-50.

Schaal, B. (2005). From amnion to colostrum to milk: Odor bridging in early developmental transitions. In: B. Hopkins \& S. Johnson (Eds), Prenatal development of postnatal functionsAdvances in infancy research. Vol. 2 (pp. 51-102). New York, NY, USA: Praeger.

Schubert, T. W. (2005). Your highness: Vertical positions as perceptual symbols of power.J. Pers. Soc. Psychol., 89, 1-21.

Sheridan, C. J., Matuz, T., Draganova, R., Eswaran, H., \& Preissl, H. (2010). Fetal magnetoencephalography-Achievements and challenges in the study of prenatal and early postnatal brain responses: A review. Infant Child Dev., 19, 80-93.

Slater, A., Morison, V., \& Rose, D. (1982). Visual memory at birth. Br. J. Psychol., 73, 519-525.

Spearman, C. (1924). The nature of "intelligence" and the principles of cognition.J. Philos., 21, 294 301. Available at https://philpapers.org/rec/SPETNO-5.

Spearman, C. (1927). The abilities of man: Their nature and measurement. London, UK: Macmillan and Co.. 
Spence, M. J. (1996). Young infants' long-term auditory memory: Evidence for changes in preference as a function of delay. Dev. Psychobiol., 29, 685-695.

Srinivasan, M., \& Carey, S. (2010). The long and the short of it: On the nature and origin of functional overlap between representations of space and time. Cognition, 116, 217-241.

Stevens, J. C., \& Marks, L. E. (1965). Cross-modality matching of brightness and loudness. Proc. Natl Acad. Sci. USA, 54, 407-411.

Streri, A. (2012). Crossmodal interactions in the human newborn: New answers to Molyneux's question. In A. J. Bremner, D. J. Lewkowicz, \& C. Spence (Eds), Multisensory development (pp. 88-112). Oxford, UK: Oxford University Press.

Streri, A., de Hevia, M. D., Izard, V., \& Coubart, A. (2013). What do we know about neonatal cognition? Behav. Sci. (Basel), 3, 154-169.

Streri, A., Lhote, M., \& Dutilleul, S. (2000). Haptic perception in newborns. Dev. Sci., 3, 319-327.

Swain, I. U., Zelazo, P. R., \& Clifton, R. K. (1993). Newborn infants' memory for speech sounds retained over 24 hours. Dev. Psychol., 29, 312-323.

VanMarle, K., \& Wynn, K. (2006). Six-month-old infants use analog magnitudes to represent duration. Dev. Sci., 9(5), F41-F49.

Van Oeffelen, M., \& Vos, P. (1982). A probabilistic model for the discrimination of visual number. Percept. Psychophys., 32, 163-170.

Walsh, V. (2003). A theory of magnitude: common cortical metrics of time, space and quantity. Trends Cogn. Sci., 7, 483-488.

Xu, F., \& Spelke, E. S. (2000). Large number discrimination in 6-month-old infants. Cognition, 74, B1-B11.

Xuan, B., Zhang, D., He, S., \& Chen, X. (2007). Larger stimuli are judged to last longer.J. Vis., 7, 2.1-5. 